\title{
Subdural Hematoma Complicating Ventriculoperitoneal Shunts: An Algerian Centers Experience
}

\author{
Ibrahim Assoumane ${ }^{1,2}$ \\ M. Al-Zekri ${ }^{1}$ \\ A. Khelifa ${ }^{1}$
}

N. Touati ${ }^{1}$

N. Lagha ${ }^{1}$

A. Sidi Said ${ }^{1}$

A. Morsli ${ }^{1}$

\begin{abstract}
Address for correspondence Ibrahim Assoumane, Department of Neurosurgery, Centre Hospitalier Universitaire, Bab El Oued, Algiers, 16000, Algeria (e-mail: as_ibrah2006@yahoo.fr; assoubrahim18@gmffail.com).
\end{abstract}

\begin{abstract}
Keywords

- subdural hematoma

- hydrocephalus

- ventriculoperitoneal shunt
\end{abstract}

Introduction Subdural hematoma can occur as a complication of a ventriculoperitoneal shunt (VPS), and it should be considered in any patient who present a shunt dysfunction or who does not show the expected recovery after revision of a shunt. Computed tomography (CT) scan is a quick and easy way of detecting subdural hematoma.

Materials and Methods We conduct a retrospective study of 17 patients. All of them are admitted for a subdural hematoma on a VPS. The diagnosis in 13 cases is made by CT scan; three cases by brain MRI.

Results We operated 16 patients and the evacuation of the hematoma with revision of the shunt was performed; we used an adjustable shunt for three patients and endoscopic third ventriculostomy in one case. The operative outcomes were favorable in 12 patients; there were 4 recurrences, 2 of which required evacuation of the hematoma through a bone flap.

Conclusion The subdural hematoma is a complication observed during the surgical treatment of the hydrocephalus and can be a serious issue. We suggest that a brain CT scan should be routinely performed in symptomatic patients with VPS.

\section{Introduction}

Subdural hematoma is one of the complications of a ventriculoperitoneal shunt (VPS) procedure, and it should be considered in any patient who presents with a malfunctioning shunt. The incidence of subdural hematoma related to overdrainage varies considerably in published reports, and appears to be approximately 3 to $12 \%{ }^{1,2}$ The subdural hematoma in shunted patients has no specific symptoms but computed tomography (CT) scan is a quick and easy way of detecting subdural hematoma.

The objective of our study was to assess the epidemiologic, clinical, and therapeutic aspects of subdural hematoma in shunted patients.

\section{Materials and Methods}

The study was conducted in the neurosurgical department of Centre Hospitalier Universitaire of Bab El Oued in Algiers,
Algeria. It was a retrospective study of 18 years from January 2000 to December 2018. We collected and studied files of 17 patients admitted for subdural hematoma on a VPS. We summarize different reasons that prompted the placement of shunt in - Table 1 .

Cervical occipito vertebral malformations are the most frequent initial pathologies found in five patients representing $29.41 \%$. The surgical technique consisted of placement of a ventriculoperitoneal fixed drainage shunt.

\section{Results}

This is a case of a patient, operated for tetraventricular hydrocephalus ( - Fig. 1) and bilateral subdural hematoma (SDH) after DVP shunt insertion ( - Fig. 2). We operated the patient and removed the SDH through burr holes and also removed the shunt ( - Fig. 3 ) and performed an endoscopic third ventriculostomy (ETV).
DOI https://doi.org/

$10.1055 / \mathrm{s}-0039-3402592$

ISSN 2277-954X. (c) 2020. Neurological Surgeons' Society of India.

This is an open access article published by Thieme under the terms of the Creative Commons Attribution-NonDerivative-NonCommercial-License, permitting copying and reproduction so long as the original work is given appropriate credit. Contents may not be used for commercial purposes, or adapted, remixed, transformed or built upon. (https://creativecommons.org/licenses/by-nc-nd/4.0/)

Thieme Medical and Scientific Publishers Pvt. Ltd., A-12, 2nd Floor, Sector 2, Noida-201301 UP, India 
During our study, we registered 17 patients admitted for a subdural hematoma on a VPS. There were 12 males $(70.60 \%)$ and 5 females $(29.40 \%)$. The sex ratio male to female (M:F) was 2.4. The mean age was 27 years with extremes from 5 months to 79 years. The childhood age and the range 17 to 40 years are the most frequent $35.29 \%$ for each ( - Table 2 ).

All 17 cases underwent VPS as initial treatment of the hydrocephalus (-Table 3). Signs of intracranial hypertension (nausea and vomitting) are the most frequent clinical signs in 13 (76.47\%) patients. The diagnosis of the subdural hematoma is made in 13 cases by CT scan and in 3 cases by brain MRI. Thirteen (76.47\%) patients presented bilateral hematoma.

We operated 16 patients and performed the evacuation of the hematoma through burr holes with revision of the shunt. We used the programmable shunt in two patients and ETV in one case, and we put one case under observation (-Table 4).

The operative outcomes were favorable in 12 patients marked by the disappearance of signs of intracranial

Table 1 Summery of different reasons that prompted the placement of shunt

\begin{tabular}{|l|l|l|}
\hline Initial pathology & Number & $\begin{array}{l}\text { Frequency } \\
\text { (\%) }\end{array}$ \\
\hline $\begin{array}{l}\text { Cervico-occipito-vertebral } \\
\text { malformations }\end{array}$ & 5 & 29.41 \\
\hline Communicanting hydrocephalus & 4 & 23.52 \\
\hline Mesencephalic tumors & 2 & 11.76 \\
\hline Pineal process & 1 & 5.88 \\
\hline Sylvius aqueductal stenosis & 1 & 5.88 \\
\hline Chronic hydrocephalus & 1 & 5.88 \\
\hline Dandy-Walker malformation & 1 & 5.88 \\
\hline Encephalopathy & 1 & 5.88 \\
\hline Post meningitis hydrocephalus & 1 & 5.88 \\
\hline Total & 17 & 100 \\
\hline
\end{tabular}

hypertension; we notice four recurrences, one of which required evacuation of the hematoma through a bone flap and programmable valve, and the other one a bone flap and ETV.

\section{Discussion}

Pathogenesis is probably not different for subdural hematoma of any etiology, but the negative ventricular pressure produced by overdrainage appears to be an important additional predisposing factor. ${ }^{2}$ Reactive subdural hematoma is believed to be the result of bleeding into the subdural space from the sudden reduction of intracranial pressure induced by cerebrospinal fluid (CSF) drainage. When the distended ventricles are drained and the intraventricular pressure is reduced, the brain tends to collapse, the subdural space widens, and disruptive traction is brought to bear upon the bridging veins. Skull enlargement, if present, exaggerates this effect because of the great discrepancy in volume between the size of the brain and the cranial cavity. ${ }^{1}$ According to Pachatouridis et $\mathrm{al}^{3}$ the valve's opening pressure is the most important factor in determining CSF drainage. This is attested by the study of Boon et al that the subdural effusions occurred in $71 \%$ of patients with a low-pressure shunt and in $34 \%$ of patients with a medium pressure shunt. ${ }^{4}$ The incidence of subdural hematoma related to overdrainage is difficult to evaluate because of lack of published series but it varies considerably in published reports. Illingworth in 1970 reported 8 cases of SDH in a series of 175 patients representing $4,5 \%^{5}$ and Samuelson et al in 1972 reported an incidence of $20 \% .{ }^{6}$ According to Boon et al, ${ }^{4}$ the incidence depends on the type of the valve and reported subdural effusions to occur in $71 \%$ of patients with a low pressure shunt and in $34 \%$ of patients with a medium pressure shunt. After using a programmable valve, Pachatouridis et $\mathrm{al}^{3}$ reported a nontraumatic subdural fluid collection (hygroma) in $12(9.4 \%)$ patients. Code et al reported an incidence of $1.4 \%$ in a series with a fixed drainage shunt. ${ }^{7}$

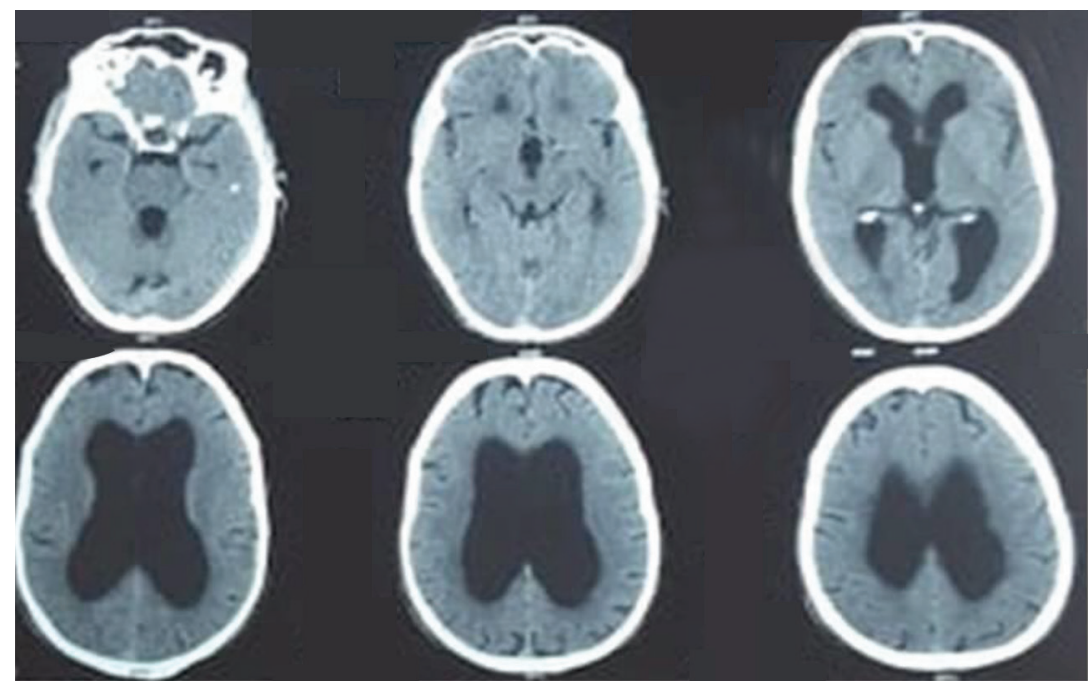

Fig. 1 Axial CT scan showing tetraventricular dilation. CT, computed tomography. 


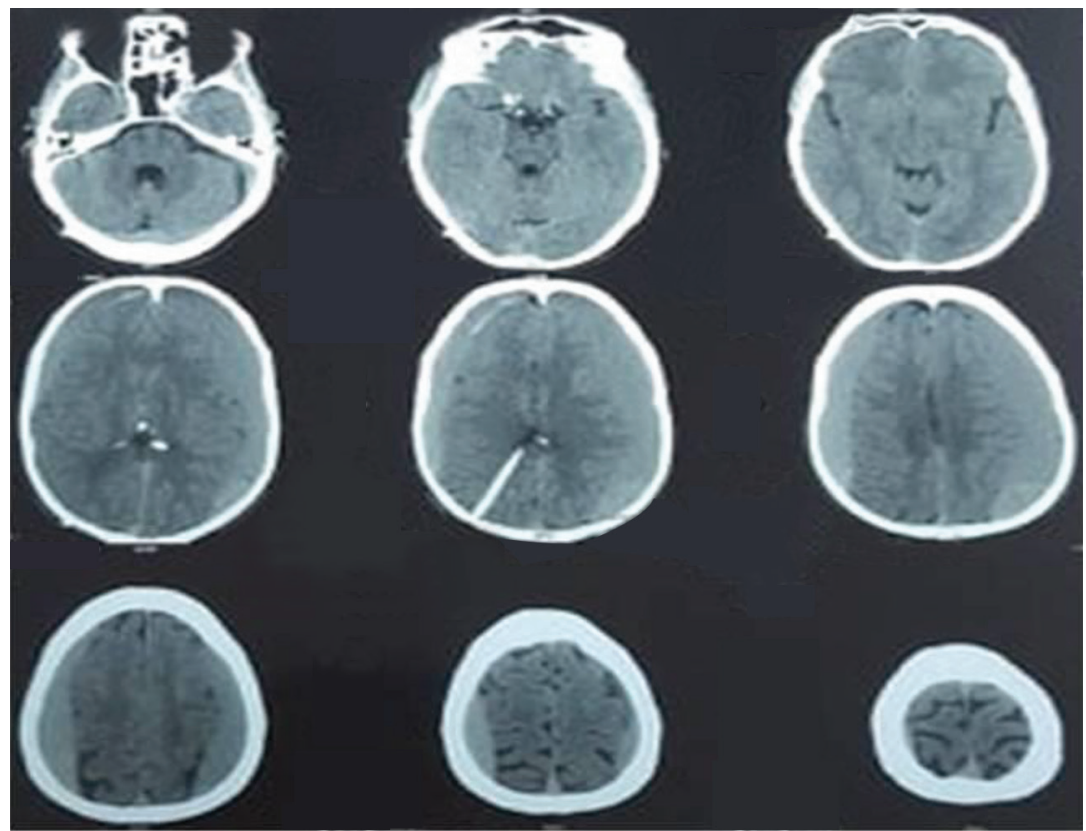

Fig. 2 Axial CT scan showing right intra ventricular shunt and bilateral subdural hematoma. CT, computed tomography.

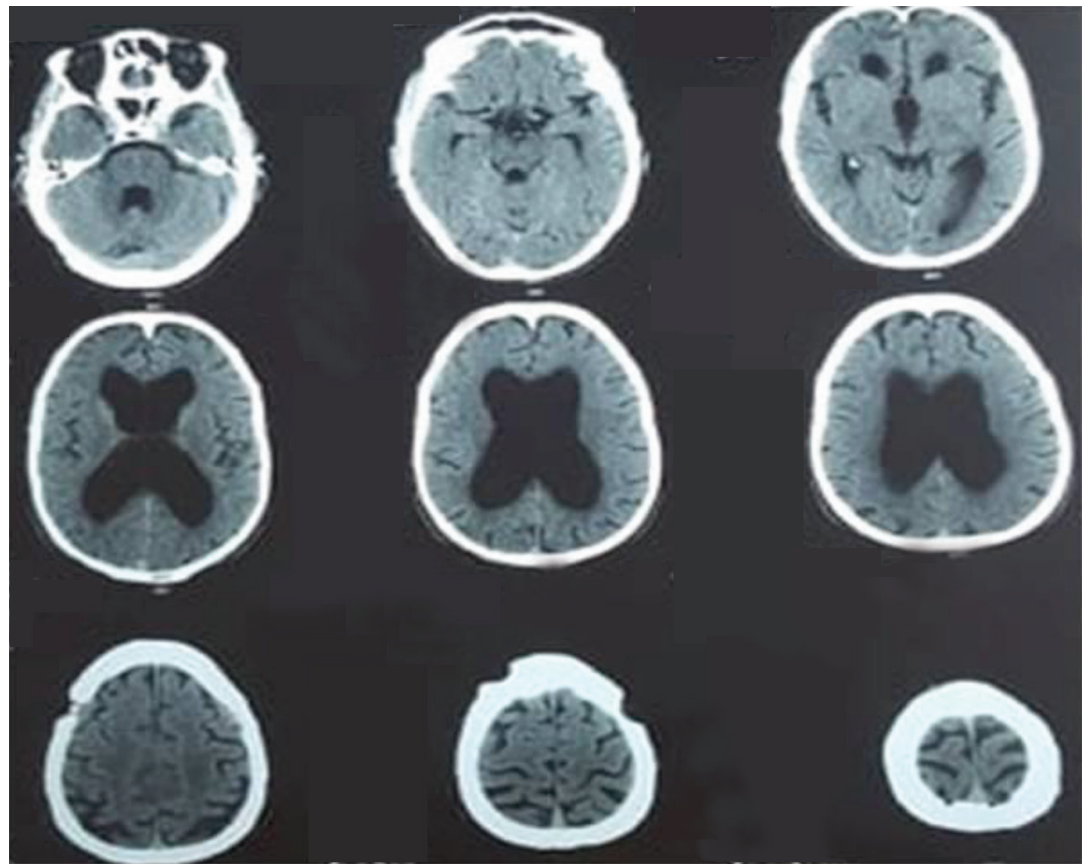

Fig. 3 Postoperative axial CT scan after evacuation of the SDH and removal of the ventricular shunt. CT, computed tomography; SDH, subdural hematoma.

Table 2 Age range of admitted patients

\begin{tabular}{|l|l|l|}
\hline Age $(\mathrm{y})$ & Number & Frequency (\%) \\
\hline $0-16$ & 6 & 35.29 \\
\hline $17-40$ & 6 & 35.29 \\
\hline $41-60$ & 3 & 17.64 \\
\hline$>60$ & 2 & 11.76 \\
\hline Total & 17 & 100 \\
\hline
\end{tabular}

Table 3 Clinical signs of the subdural hematoma

\begin{tabular}{|l|l|l|}
\hline Clinical signs & Number & Frequency (\%) \\
\hline Intracranial hypertension & 13 & 76.47 \\
\hline Cerebellar syndrome & 2 & 11.76 \\
\hline Pyramidal tract syndrome & 1 & 5.88 \\
\hline Macrocranie & 1 & 5.88 \\
\hline Total & 17 & 100 \\
\hline
\end{tabular}


Table 4 Treatment of the hematoma and the hydrocephalus

\begin{tabular}{|l|l|l|}
\hline $\begin{array}{l}\text { Treatment of hematoma and } \\
\text { hydrocephalus }\end{array}$ & Number & $\begin{array}{l}\text { Frequency } \\
\text { (\%) }\end{array}$ \\
\hline Burr holes and valve revision & 13 & 76.47 \\
\hline Burr holes and programmable valve & 2 & 11.76 \\
\hline Burr holes and ETV & 1 & 5.88 \\
\hline Observation & 1 & 5.88 \\
\hline Total & 17 & 100 \\
\hline
\end{tabular}

Abbreviation: ETV, endoscopic third ventriculostomy.

In our series, cervical occipito vertebral malformations are the most frequent cause of hydrocephalus representing $29.41 \%$ followed by communicanting hydrocephalus $23.52 \%$. Subdural hematomas in shunted patients do not cause specific symptomatology. While some subdural collections are benign and can resolve spontaneously, others may grow to cause significant mass effect and neurologic compromise. ${ }^{8}$ The most frequent clinical signs are signs of intracranial hypertension in $76.47 \%$ of patients, followed by cerebella syndrome in two patients representing $11.76 \%$ of patients.

We operated 16 patients and performed the evacuation of the hematoma through burr holes with revision of the shunt in 13 patients (76.47\%); for two patients, after the evacuation of the hematoma through burr holes, we changed the shunt to programmable valve. In the series reported by Boon et al, most of the patients were asymptomatic and resolved spontaneously; only approximately $16 \%$ of the subdural collections that formed required surgical drainage; 4 we put one patient under observation and the hematoma resolved spontaneously.

The advancement of adjustable shunt brings many advantages; Fukuhara et al supported that there have been cases reported in which adjustment of the shunt valve alone allowed for complete resolution of postshunt SDHs. ${ }^{9}$

The significantly high proportion of noninvasive treatment in the group with adjustable shunts also indicates that if patients with fixed valves had received adjustable shunts instead it is most likely that many surgical procedures could have been avoided. ${ }^{10}$

The postoperative outcome was favorable in 12 patients marked by the disappearance of signs of intracranial hypertension, but we noticed four recurrence cases. Although not significant, the mortality rate at 1 year, after a complication, was higher in the patient group undergoing surgery than in the group treated with opening pressure adjustments. This suggests that a nonsurgical treatment could be preferable than a surgical one. ${ }^{10}$

\section{Conclusion}

The subdural hematoma is a complication observed during the surgical treatment of the hydrocephalus and can be a fatal complication. We suggest that a brain CT scan should be routinely performed in symptomatic patients with VPS. Use of the newer programmable shunt can help reduce the problem of over functioning VPS and its related complications.

\section{Conflict of interest}

None declared.

\section{References}

1 Moussa AH, Sharma SK. Subdural haematoma and the malfunctioning shunt. J Neurol Neurosurg Psychiatry 1978;41(8): 759-761

2 Sternbach GL, Sternbach MS. Subdural hematoma in a shunted patient. J Emerg Med 2005;29(4):483-484

3 Pachatouridis D, Alexiou GA, Mihos E, Fotakopoulos G, and Voulgaris S. The value of programmable shunt valves for the management of subdural collections in patients with hydrocephalus. Sci World J 2013;2013:461896

4 Boon AJ, Tans JT, Delwel EJ, et al. Dutch Normal-Pressure Hydrocephalus Study: randomized comparison of low- and medium-pressure shunts. J Neurosurg 1998;88(3):490-495

5 Illingworth RD. Subdural haematoma after the treatment of chronic hydrocephalus by ventriculocaval shunts. J Neurol Neurosurg Psychiatry 1970;33(1):95-99

6 Samuelson S, Long DM, Chou SN. Subdural hematoma as a complication of shunting procedures for normal pressure hydrocephalus. J Neurosurg 1972;37(5):548-551

7 Code BM, Aissata LB, Katanga BA, Abdoukarim O, Ndaraw N, Badara TA, Youssoupha S, Boubakar BS. Hématome sous dural, complication du traitement chirurgical des hydrocéphalies. Notre experience a dakar. African J Neurol Sci 2005;24(2)28-32

8 Paff M, Alexandru-Abrams D, Muhonen M, Loudon W. Ventriculoperitoneal shunt complications: a review. Interdiscip Neurosurg 2018;13:66-70

9 Fukuhara T, Vorster SJ, Luciano MG. Critical shunt-induced subdural hematoma treated with combined pressure-programmable valve implantation and endoscopic third ventriculostomy. Pediatr Neurosurg 2000;33(1):37-42

10 Sundström N, Sundström N, Lagebrant M, Eklund A, Koskinen LD, Malm J. Subdural hematomas in 1846 patients with shunted idiopathic normal pressure hydrocephalus: treatment and long-term survival. J Neurosurg 2018;129(3):797-804 